\title{
ACM Transactions on Human-Robot Interaction: The State of the Journal
}

As the first robotics journal offered by ACM, the Transactions on Human-Robot Interaction (ACM THRI) made considerable progress to advance the field of HRI since its inaugural offering in 2018. ACM THRI remains the flagship journal for leading research in the burgeoning area of Human-Robot Interaction (HRI). The interdisciplinary field of HRI aims to understand and advance our knowledge of human-robot systems towards advancing usable and useful robotic technology. ACM THRI features leading thought into how people interact with robots and robotic technologies, how to improve these interactions and make new kinds of interaction possible, and the effects of such interactions on organizations or society. HRI brings together researchers and practitioners with diverse expertise, including but not limited to robotics, artificial intelligence, human factors, psychology, design, haptics, and mechatronics.

We officially began our term in January 2017 as Editors-in-Chief of the Journal of Human-Robot Interaction, the predecessor to ACM THRI. We were fortunate to build on the foundation of JHRI provided by its founding editors, Profs. Sara Kiesler and Michael Goodrich. Our leadership of ACM THRI would not be possible without the tremendous effort and dedication of our core editorial team, including our amazing Managing Editor (Prof. James Young), Journal Administrator (Gita Delsing) and Information Director (Dr. Daniel Rea) as well as their predecessors (Prof. David FeilSeifer, Ms. Jeanie Lyubelsky, and Prof. Monica Anderson). Although it has presented unique challenges and new opportunities, we remain grateful for the opportunity to lead the transition of JHRI to ACM ownership and further extend ACM THRI.

Our focus as Editors-in-Chief has been to establish commitment to excellence, sustainability, and inclusion in the scholarship and review processes of ACM THRI, which has proved successful to this point. As stated in our introductory editorial, our primary aims for the Journal have been three fold: (1) increase the sustainability and impact of HRI as a field (both quantitatively and qualitatively), (2) enable timely and productive review feedback, and (3) cultivate new and leading ideas in both robotics and the human-centered sciences. ACM THRI has remained true to this scope and made significant strides towards realizing these values across the practices of the journal.

For our review processes, our goal has been to provide useful feedback to our authors that enables them to improve their work and make contributions worthy of intellectual leadership. We aim to return substantive review feedback to authors within 105 days of the date of submission, and 70 days for revisions. The total average for ACM THRI returning decisions for submitted manuscripts is currently 142 days in 2021, 122 days from April 2020 to December 2020, 114.7 days in the first three months of 2020 (submissions just prior to the COVID-19 pandemic), and 99 days in 2019. Our review process includes significant deliberation and discussion among our editorial board of every manuscript and decision, which occurs during our weekly editorial teleconferences. A summary of our editorial consensus with a reasoned and supported argument is provided with

\footnotetext{
ACM Reference format: https://doi.org/10.1145/3488567

(C) 2022 Copyright held by the owner/author(s).

2573-9522/2022/02-ART1e

https://doi.org/10.1145/3488567
}

Selma Šabanović and Odest Chadwicke Jenkins. 2022. ACM Transactions on Human-Robot Interaction: The State of the Journal. ACM Trans. Hum.-Robot Interact. 11, 1, Article 1e (February 2022), 3 pages. 
the decision notification of each letter. We, as Editors-in-Chief, make ourselves available to authors to answer any questions they have about review feedback, our review processes, and publication practices. We have received positive feedback from authors and associate editors on the quality of the review process and care with which papers are handled.

During our term as Editors-in-Chief, we are seeing growth in our field and the Journal over the four volumes we have published (one as JHRI and four as ACM THRI). During our term as Editors-in-Chief, we have overseen the growth in the number of issues published per volume (from 3 to 4 per year) and the number of articles published per issue (from 4 in the beginning to 10 at the moment). Under our leadership, ACM THRI has gained indexing by SCOPUS and is under review for indexing by Clarivate Analytics. Our publications of ACM THRI feature a combination of opinion leaders in HRI research, emerging early career scholars, and leading figures from a broader interdisciplinary community. We have made a point to be inclusive in our intellectual perspective for the journal, and to serve as a less time-limited complement to the groundbreaking ACM/IEEE HRI Conference.

We are also grateful for the opportunity to work collaboratively as Editors-in-Chief to cultivate new, diverse, and substantive ideas to chart the intellectual future of HRI. Our own work contributes to and is informed by different perspectives on HRI - the more computational/technical and the more social/design oriented - and we feel that having in-depth knowledge of diverse scientific practices and literatures that contribute to HRI allows us to nurture a broader array of work in the journal. We similarly have made sure to solicit scholars with diverse intellectual backgrounds for the journals' Editorial Board, which contributes to our ability to evaluate manuscripts based on the contributions that are significant for their specific fields. One of our most important innovations has been to establish sections of the journal to address different dimensions of HRI research: Behavioral/Social, Computational, Design, and Mechanical. Each of these sections is led by a pair of Senior Associate Editors. In addition to providing greater interdisciplinary inclusion, we believe this multiresolutional model will be essential for scalability as the field of HRI grows and the number of submitted manuscripts increases. We believe enabling greater autonomy for the Senior Associate Editor roles will be needed in the near future to help manage growth and strengthen interdisciplinarity in the journal.

To further stimulate a diverse and interdisciplinary authorship and readership for the journal, we regularly feature Special Issues on novel topics in HRI. So far, we have published five special issues at the rate of about one per year and have two more under development. The Special Issues cover the following themes: Artificial Intelligence for HRI (published 2018); Representation Learning for Human and Robot Cognition (published 2019); Explainable Robot Behavior (to be published 2021); Test Methods for Human-Robot Teaming Evaluations (to be published 2022), and Designing the Robot Body: Critical Perspectives on Affective Embodied Interaction (to be published 2022). We are incredibly appreciative to the guest editors for these special issues for their considerable time and effort to cultivate new frontiers for our field. From an organizational perspective, we are actively working to improve our process to improve the experience for guest editors and submitting authors to special issues.

Our term also introduced a Perspectives category for submissions, which are editorial style short papers on a particular topic solicited by the EiCs or volunteered by authors. We published a series of Perspectives in our first issue, and have had several other Perspectives articles submitted recently. We strive to solicit Perspectives articles more regularly, and the journal continually remains open to receiving such submissions from scholars across the spectrum of HRI. One particular topic of discussion across HRI in recent years has been on how we should think about empirical methods as a community. Given this discussion, we were thrilled to publish a set of Perspectives articles on Empirical Methods in HRI, connected with an accepted article. We aspire to 
do more similar targeted perspectives collections in the future. Also falling under the domain of future aspiration for ACM THRI, a section of the journal on philosophy, ethics, and policy for HRI is a clear objective on the foreseeable horizon.

In our second term, we aim to build on the strengths of the Journal (e.g., disciplinary and geographic inclusiveness, timely and high-quality review), and work on some of the challenges we experienced in our first term (e.g., associate editor engagement and workload, indexing). Our main aims are to improve scalability, breadth, and outreach to new audiences. We have already moved forward with our plan to strengthen our editorial board by inviting new members, and incorporating new subfields of HRI as needed. This will support our aim of sustaining and even increasing the interdisciplinary reach of the journal. We will also work with Senior Associate Editors to increase their autonomy in decision-making, which fits our aims to further grow the number of submissions to the journal. We will continue to bring SAEs together through regular teleconferences with the EiCs and Managing Editor to discuss journal priorities and comment on processes. We are also working to be more visible on popular and social media to improve the visibility of the Journal and maximize the impact of our authorship.

Lastly, we must thank ACM Publications for their great support and responsiveness. Laura Lander has been a joy as our primary interface to ACM. She has been a tremendous resource in our startup mode and transition to ACM. We are especially grateful for her connecting us to our current Journal Administrator, Gita Delsing, who has been essential to our smooth operations over the past two years.

\author{
Selma Šabanović \\ Indiana University \\ Odest Chadwicke Jenkins \\ University of Michigan \\ Co-Editors-in-Chief
}

